\title{
Supersolidity from defect condensation in the extended boson Hubbard model
}

\author{
Yu-Chun Chen, ${ }^{1}$ Roger G. Melko, ${ }^{2,3}$ Stefan Wessel, ${ }^{4}$ and Ying-Jer Kao ${ }^{1,5, *}$ \\ ${ }^{1}$ Department of Physics, National Taiwan University, Taipei 106, Taiwan \\ ${ }^{2}$ Department of Physics and Astronomy, University of Waterloo, Ontario, Canada N2L $3 G 1$ \\ ${ }^{3}$ Materials Science and Technology Division, Oak Ridge National Laboratory, Oak Ridge, Tennessee 37831, USA \\ ${ }^{4}$ Institut für Theoretische Physik III, Universität Stuttgart, 70550 Stuttgart, Germany \\ ${ }^{5}$ Center for Theoretical Sciences, National Taiwan University, Taipei 106, Taiwan
}

(Received 26 October 2007; published 28 January 2008)

\begin{abstract}
We study the ground-state phase diagram of the hard-core extended boson Hubbard model on the square lattice with both nearest- and next-nearest-neighbor hoppings and repulsions, using Gutzwiller mean-field theory and quantum Monte Carlo simulations. We observe the formation of supersolid states with checkerboard, striped, and quarter-filled crystal structures when the system is doped away from commensurate fillings. In the striped supersolid phase, a strong anisotropy in the superfluid density is obtained from the simulations; however, the transverse component remains finite, indicating a true two-dimensional superflow. We find that upon doping, the striped supersolid transitions directly into the supersolid with quarter-filled crystal structure, via a first-order stripe melting transition.
\end{abstract}

DOI: 10.1103/PhysRevB.77.014524

PACS number(s): 74.20.-z, 75.10.Jm, 05.30.Jp, 67.25.D-

\section{INTRODUCTION}

In 1956, Penrose and Onsager ${ }^{1}$ first posed the question of whether one could expect superfluidity in a solid-a supersolid state-with coexisting diagonal and off-diagonal longrange orders. They showed that for a perfect crystal, where the wave function of the particles is localized near each lattice site, superfluidity does not occur at low temperature. Later, it was proposed ${ }^{2-4}$ that fluctuating defects in imperfect crystals can condense to form a superfluid, and a supersolid state (with both superflow and periodic modulation in the density) emerges. In 2004, Kim and Chan reported signatures of superfluidity in solid ${ }^{4} \mathrm{He}$ in torsional oscillator experiments, ${ }^{5}$ where a drop in the resonant period, observed at around $T \sim 0.2 \mathrm{~K}$, suggested the existence of a nonclassical rotational inertia in the crystal. ${ }^{4}$ Following the discovery by Kim and Chan, many experiments and theories have attempted to explain this fascinating observation; the situation remains, however, controversial. ${ }^{6-11}$

On the other hand, with improvements of quantum Monte Carlo (QMC) methods, the origin of supersolid phases can be studied exactly in both continuum and lattice models. Exotic quantum phases, including supersolids, are highly sought after in lattice models, particularly those that may be realized by loading ultracold bosonic atoms onto optical lattices. ${ }^{12,13}$ The generation of a Bose-Einstein condensate in a gas of dipolar atoms ${ }^{14}$ with longer-range interactions provides one promising route to search for the supersolid state. ${ }^{15}$ The extended boson Hubbard model is the obvious microscopic Hamiltonian to study these systems, and supersolids have been found in this model on various lattices. ${ }^{16-20} \mathrm{~A}$ simplified phenomenological picture for understanding the supersolid phase in these models has been the aforementioned "defect-condensation" scenario: starting from a perfect lattice crystal at commensurate filling, supersolidity arises when dopants (particles or holes) condense and contribute a superflow. In the simplest scenario-hard-core bosons doped above commensurate filling - this phenomenological picture suggests "microscopic phase separation" be- tween the crystal and superfluid sublattices. Recent work on triangular lattice supersolids ${ }^{21,22}$ has called this simple interpretation into question, since there one apparently finds examples where particles on the crystal lattice also take part in the superflow. However, frustration complicates the interpretation of these results, since the underlying order-by-disorder mechanism facilitates supersolid formation at half-filling.

To more closely study the degree to which defect condensation plays a role in the mechanism behind lattice supersolids, we study the extended hard-core boson Hubbard model with both nearest-neighbor (nn) and next-nearest-neighbor (nnn) hopping and repulsive interactions on the square lattice. The Hamiltonian is

$$
\begin{aligned}
\mathcal{H}= & -t \sum_{\langle i, j\rangle}\left(a_{i}^{\dagger} a_{j}+a_{i} a_{j}^{\dagger}\right)+V_{1} \sum_{\langle i, j\rangle} n_{i} n_{j}-\mu \sum_{i} n_{i}-t^{\prime} \sum_{\langle\langle i, j\rangle\rangle}\left(a_{i}^{\dagger} a_{j}\right. \\
& \left.+a_{i} a_{j}^{\dagger}\right)+V_{2} \sum_{\langle i, j\rangle\rangle} n_{i} n_{j},
\end{aligned}
$$

where $a_{i}^{\dagger}$ and $a_{i}$ are the boson creation and annihilation operators, $n_{i}=a_{i}^{\dagger} a_{i}$ is the number operator, and $\langle i, j\rangle$ denotes the nearest- and $\langle\langle i, j\rangle\rangle$ next-nearest neighbors. The $t^{\prime}=0$ limit has been studied previously, and is known to harbor several crystal solids, including a checkerboard structure that upon doping is unstable toward phase separation. ${ }^{18,23}$ A striped crystal and a stable striped supersolid were also found in the $t^{\prime}=0$ limit. ${ }^{18}$

Here, we study the full Hamiltonian of Eq. (1) using both Gutzwiller mean-field theory (Sec. II) and stochastic series expansion QMC simulations (Sec. III) based on the directed loop algorithm. ${ }^{24,25} \mathrm{We}$ confirm that the model contains a variety of lattice crystals, including a checkerboard and striped phase at half-filling, plus a quarter-filled solid. ${ }^{26}$ The nnn hopping $t^{\prime}$ is found to stabilize a checkerboard supersolid away from half-filling; this and other supersolid phases with differently broken symmetry are studied in detail. In general, we find that although supersolid phases are not stabilized at commensurate fillings, they are readily formed upon doping. However, we demonstrate that, contrary to the 

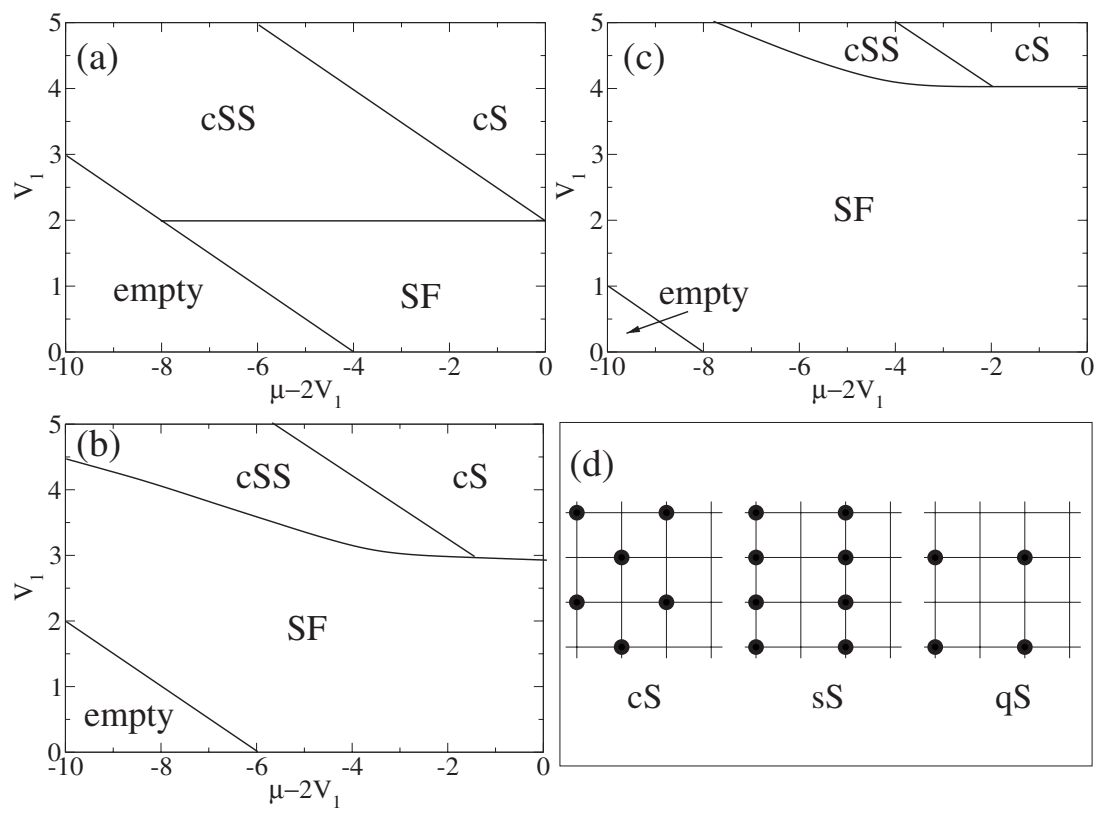

FIG. 1. Mean-field phase diagram of $t-t^{\prime}$ $-V_{1}$ model at (a) $t=0$, (b) $t=0.5$, and (c) $t=1.0$. All transitions are continuous. See text for discussion of the phases. (d) Possible quantum solid configurations: checkerboard solid (cS), striped solid (sS), and quarter-filled solid (qS). All quantities are in units of $t^{\prime}$. simple phenomenological picture of defect condensation, where the crystal and superfluid sublattices are clearly distinct, in at least one of our supersolid phases, particles from the crystal sublattice also participate to a large degree in the superflow.

\section{GUTZWILLER MEAN-FIELD APPROXIMATION}

We begin by surveying the ground-state phase diagram of the model in several limits using Gutzwiller mean-field theory. The Gutzwiller variational $\operatorname{method}^{27}$ is a powerful technique for studying strongly correlated system. The ground state of an interacting system is constructed from the corresponding noninteracting ground state,

$$
\left|\phi_{g}\right\rangle=\prod_{i}\left(\sum_{n_{i}} f_{n_{i}}\left|n_{i}\right\rangle\right) .
$$

Here, the $f_{n_{i}}$ are site-dependent variational parameters, which can be optimized via minimizing the energy,

$$
E_{0}=\frac{\left\langle\phi_{g}|\mathcal{H}-\mu N| \phi_{g}\right\rangle}{\left\langle\phi_{g} \mid \phi_{g}\right\rangle},
$$

of the variational state. The $\left|n_{i}\right\rangle$ form the local Fock basis at site $i$ with $n_{i}$ particles in state $\left|n_{i}\right\rangle$. In the hard-core limit, we only need to keep the local states $|0\rangle$ and $|1\rangle$. Physical quantities are calculated within the variational ground states. In particular, we measure the local density $\left\langle n_{i}\right\rangle$, the density structure factor at wave vector $\mathbf{q}$,

$$
S(\mathbf{q})=\frac{1}{N} \sum_{i, j} e^{i \mathbf{q} \cdot\left(\mathbf{r}_{i}-\mathbf{r}_{j}\right)}\left\langle n_{i} n_{j}\right\rangle,
$$

and superfluidity due to a finite value of $\left\langle a_{i}\right\rangle$. The coexistence of superfluid order and Bragg peaks in the structure factor signifies supersolidity.

We begin by studying the phase diagram within the Gutzwiller approximation near half-filling in the absence of the nnn repulsion (i.e., for $V_{2}=0$ ). From Fig. 1, we find that at the mean-field level, various phases are stabilized already by this restricted set of parameters. These include a uniform superfluid (SF), a checkerboard solid (cS) with ordering wave vector $\mathbf{q}=(\pi, \pi)$ [see Fig. 1(d) for an illustration], and, in particular, a checkerboard supersolid (cSS) — with coexisting diagonal order and superfluidity, away from half-filling $\left(\mu=2 V_{1}\right)$.

As expected, increasing the nn hopping $t$ destroys the solidity of the system. In particular, the supersolid region, found when the system is doped away from half-filling, becomes a uniform superfluid at large $t$. This clearly indicates that large $t$ destabilizes the supersolid state.

To study the effects of a finite nnn repulsion $V_{2}$, we first identify two limiting cases. For the $t-V_{2}$ model, a stable supersolid state is the striped supersolid $(\mathrm{sSS})^{16,17,23,28}$ with ordering wave vectors $\mathbf{q}=(\pi, 0)$ or $(0, \pi)$ if obtained. For the $t^{\prime}-V_{1}$ model, a stable supersolid state is the checkerboard supersolid. To capture the behavior of the system between these limiting regimes, we introduce a parameter $0 \leqslant x \leqslant 1$, which interpolates between the two regions, by setting $t=x$, $t^{\prime}=1-x$, and $V_{2}=5 x$ (see Ref. 26). In the following, we thus work in units of $t+t^{\prime}=1$. Figure 2 shows Gutzwiller meanfield phase diagrams for different values of $x=0,0.5$, and 1 . While for finite $V_{2}$, half-filling is obtained for $\mu=2 V_{1}+2 V_{2}$, we still take $\mu-2 V_{1}$ as the abscissa, in order to ease a direct comparison to the previous case of $V_{2}=0$.

The coexistence of both $\mathrm{nn}$ and nnn repulsions is expected to stabilize various solid states: ${ }^{26}$ at half-filling, with both $V_{1}$ and $V_{2}$ large, a checkerboard (striped) solid is formed for $V_{1}>2 V_{2}\left(V_{1}<2 V_{2}\right) ;{ }^{16}$ we find that for $\rho=1 / 4$, a quarter-filled solid (qS) [shown in Fig. 1(d)] emerges. In order to distinguish the different solids, we measure the structure factors at reciprocal lattice vectors $\mathbf{q}=(\pi, \pi),(\pi, 0)$, and $(0, \pi)$. In addition, for the striped structure where a strong anisotropy due to the broken rotational symmetry occurs, the magnitude of the difference between $O_{x}=S(\pi, 0)$ and $O_{y}=S(0, \pi)$ is almost 

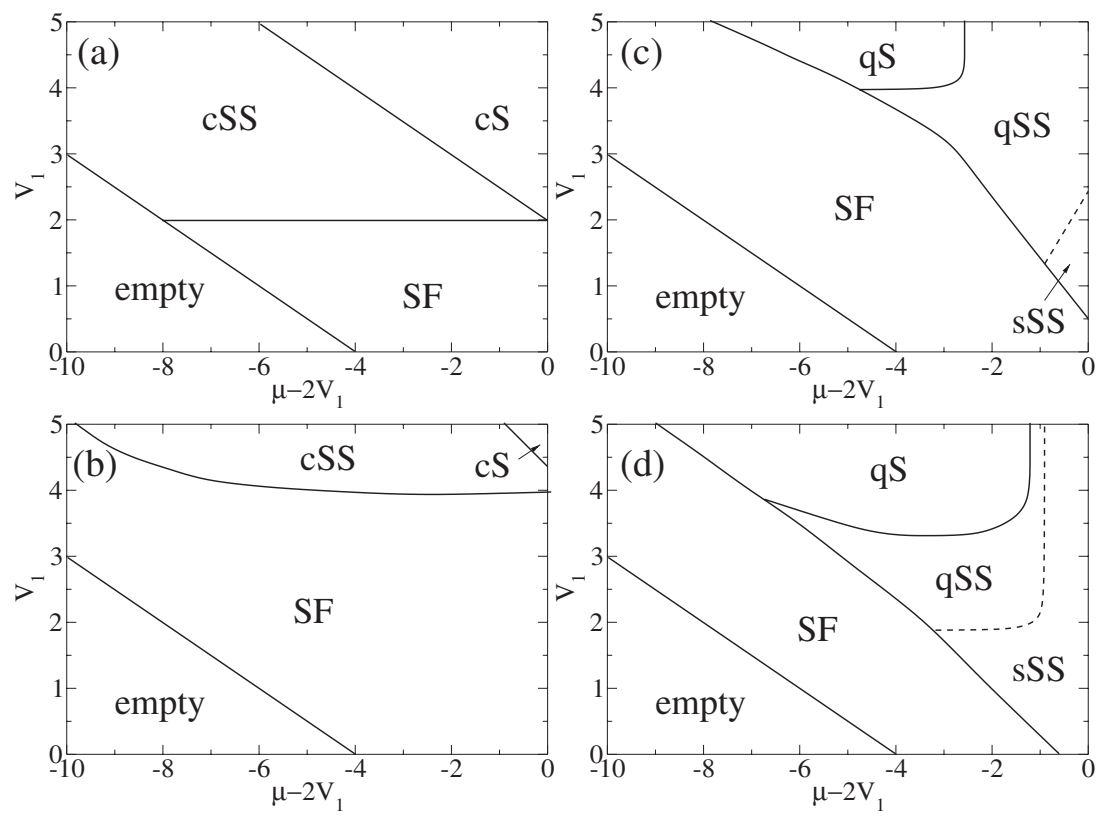

FIG. 2. Mean-field phase diagram of $t-t^{\prime}$ $-V_{1}-V_{2}$ model at (a) $x=0: t=0, t^{\prime}=1.0$, and $V_{2}$ $=0$, (b) $x=0.3: t=0.3, t^{\prime}=0.7$ and $V_{2}=1.5$, (c) $x$ $=0.7: t=0.7, t^{\prime}=0.3$ and $V_{2}=3.5$ and (d) $x=1.0: t$ $=1.0, t^{\prime}=0$ and $V_{2}=5$. Solid lines indicate continuous transitions and dashed lines indicate firstorder transitions. All quantities are in units of $t$ $+t^{\prime}$. equal to the sum $O_{x}+O_{y}$. Keeping track of this quantity allows us to easily distinguish supersolids with an underlying striped crystal, from supersolids with an underlying quarterfilled crystal, in which case all three structure factors become finite, but the difference $\left|O_{x}-O_{y}\right|$ is zero (see Table I for a summary).

The case $x=0$, shown in Fig. 2(a), corresponds to the $t^{\prime}$ $-V_{1}$ model, which we already discussed [compare to Fig. 1(a)]. At $x=0.3$ [Fig. 2(b)], the superfluid phase expands, as the introduction of $t$ and $V_{2}$ destabilizes the checkerboard solid. For $x=0.7$ [Fig. 2(c)], with the model parameters approaching the $t-V_{2}$ limit, the checkerboard structure disappears. Instead, striped and quarter-filled structures emerge, including a sSS and a quarter-filled supersolid (qSS). ${ }^{29}$ In the limiting case $x=1$ [Fig. 2(d)] we find two different transition paths from the sSS to the superfluid upon doping. When $V_{1}$ $<2$, the striped supersolid enters the superfluid directly. When $V_{1}>2$, the qS and qSS regions are passed as an intermediate regime, separating the sSS and the superfluid.

Clearly, these mean-field phase diagrams provide evidence for not only the existence of various different supersolid phases but also for the possibility of direct quantum phase transitions between them (in particular, between the qSS and sSS phases). In the next section, using these meanfield results as guidance, we turn to quantum Monte Carlo simulations in order to study in detail the various supersolid phases, as well as the transitions between them.

TABLE I. Order parameters associated with the different crystal orders.

\begin{tabular}{lccc}
\hline \hline Structure factor & Checkerboard & Striped & Quarter Filled \\
\hline$S(\pi, \pi)$ & $\neq 0$ & 0 & $\neq 0$ \\
$O_{x}+O_{y}$ & 0 & $\neq 0$ & $\neq 0$ \\
$\left|O_{x}-O_{y}\right|$ & 0 & $\simeq O_{x}+O_{y}$ & 0 \\
\hline \hline
\end{tabular}

\section{QUANTUM MONTE CARLO RESULTS}

We performed extensive QMC simulations of the Hamiltonian Eq. (1) using a variation of the stochastic series expansion framework with directed loops. ${ }^{24,25}$ Correlation functions of density operators are easily measured within the QMC, and crystal order is signified by peaks in the q-dependent structure factor of Eq. (3). The superfluid density is measured in the standard way in terms of winding number fluctuations,

$$
\rho_{s}^{\mathbf{a}}=\frac{\left\langle W_{\mathbf{a}}^{2}\right\rangle}{\beta},
$$

where a labels the $x$ or $y$ direction and $\beta$ is the inverse temperature. Typically, the stiffness is averaged over both directions, unless measured in a striped phase which breaks rotational symmetry (as discussed below). In the following, we choose $\beta$ large enough to ensure simulation of groundstate properties, and the system size is $N=L \times L$.

We begin by examining the phase transition into the cSS state, identified in Fig. 1. In the limit where $V_{2}$ vanishes, Fig. 3 shows the behavior of the QMC observables at $V_{1}=3.5 t^{\prime}$. For $t \gg t^{\prime}\left(t=100 t^{\prime}\right)$ (open symbols), there is a discontinuity near $\mu-2 V_{1}=-5 t^{\prime}$, where a checkerboard solid with finite $S(\pi, \pi)$ melts into a superfluid with finite $\rho_{s}$ via a first-order transition. This discontinuous jump in the particle density near $\mu-2 V_{1}=-5 t^{\prime}$ is a clear indication that phase separation would occur in a canonical system. ${ }^{23}$ In contrast, in the limit $t \ll t^{\prime}\left(t=0.01 t^{\prime}\right)$ (solid symbols), the discontinuity disappears, and a smooth decrease in the structure factor as holes are doped into the system is accompanied by an increasing superfluidity. The coexistence of both finite $S(\pi, \pi)$ and $\rho_{s}$ in contrast to the case $t^{\prime}=0$ indicates that a checkerboard supersolid state is stabilized by the nnn hopping. In order to confirm that this is indeed true, we perform simulations with finite nn hopping $t$. In Fig. 4, we show QMC results as a function of $\mathrm{nn}$ hopping $t$ for various $V_{1}$ and the chemical 


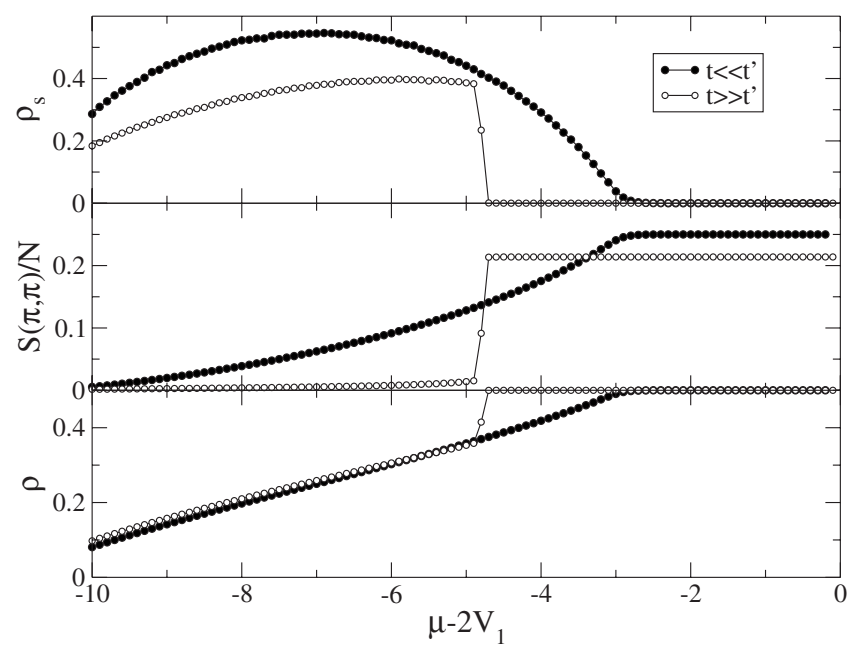

FIG. 3. $\rho_{s}, S(\pi, \pi)$, and the number density $\rho$ vs the chemical potential $\mu$ for $V_{1}=3.5$ and $L=16$ at an inverse temperature $\beta=10$. All quantities are in units of $t^{\prime}$.

potential fixed at $\mu-V_{1}=-3.5 t^{\prime}$. A supersolid phase emerges for $V_{1}>2.5 t^{\prime}$, and a checkerboard supersolid to superfluid transition occurs as $t$ increases. The smooth nature of the data across the transition region suggests that the destabilization of the cSS state upon increased $t$ occurs via a continuous phase transition.

Next, we consider the effect of the nnn repulsion $V_{2}$, as alluded in Fig. 2. We focus on the results from simulations performed at $x=0.9$, corresponding to $t=0.9, t^{\prime}=0.1$, and $V_{2}=4.5$. Three different values of the nn repulsion are chosen: $V_{1}=1.0,3.0$, and 4.5. For $V_{1}=1.0$, the dominant $t$ and $V_{2}$ render the model close to a $t-V_{2}$ model, and a striped structure is expected (Fig. 2). In Fig. 5, the equivalence of $O_{x}$ $+O_{y}$ and $\left|O_{x}-O_{y}\right|$ indicates the absence of the quarter-filled structure, and indeed, at half-filling, a stable striped solid

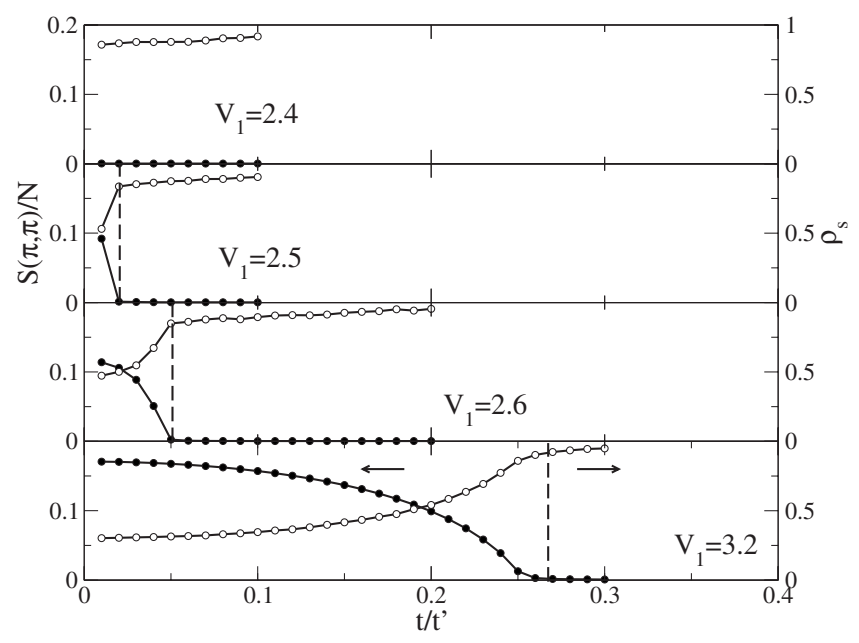

FIG. 4. $S(\pi, \pi)$ (solid symbols) and $\rho_{s}$ (open symbols) vs the nn hopping $t . \mu-2 V_{1}=-3.5, \beta=10$, and $L=32$. The vertical dash line divides the two phases: the right-hand side is a superfluid state and the left-hand side is a checkerboard supersolid state. All quantities are in units of $t^{\prime}$.

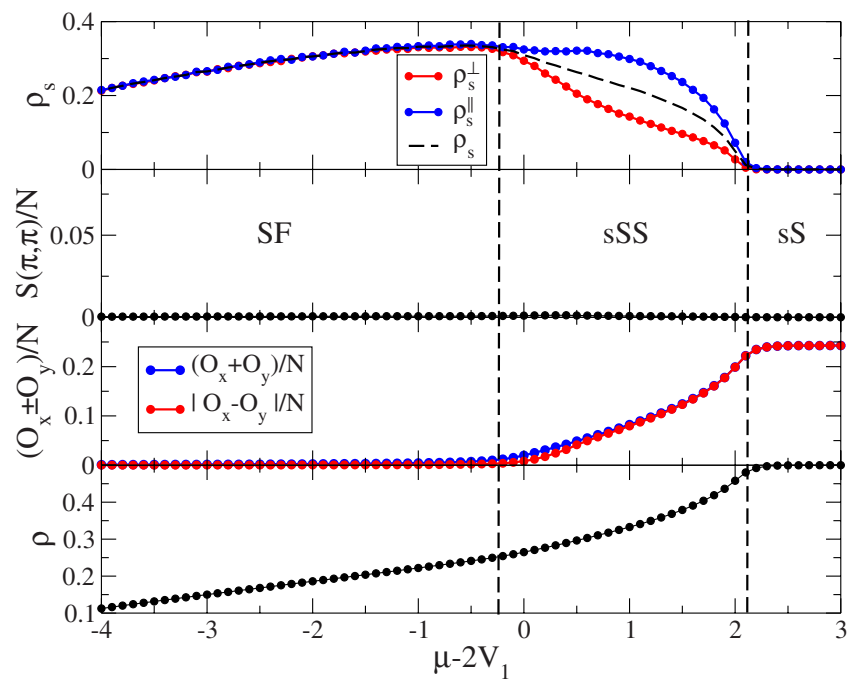

FIG. 5. (Color online) The structure factors $\rho_{s}$ and $\rho$ for $x=0.9$, $V_{1}=1.0, L=16$, and $\beta=10$. In the striped supersolid (sSS), $\rho_{s}$ shows a strong anisotropy. All quantities are in units of $t+t^{\prime}$.

(sS) is formed. Furthermore, upon hole doping away from $\rho=1 / 2$, a striped supersolid emerges. To assess the behavior of the superflow in the sSS, we measured the superfluid densities perpendicular and parallel to the actual stripe direction. For this purpose, $\rho_{s}^{\perp}$ and $\rho_{s}^{\|}$are defined by comparing the magnitude of $O_{x}$ and $O_{y}$ calculated after each Monte Carlo step: when $O_{x}>O_{y}$, the $x$-direction winding number $W_{x}$ [see Eq. (4)] is counted as $W_{\|}$and $W_{y}$ is counted as $W_{\perp}$, and vice versa. ${ }^{21}$ Figure 5 clearly exhibits a pronounced anisotropy of $\rho_{s}$ in the sSS phase. Upon further hole doping, we observe a melting of the crystal structure to a uniform SF. This completes the quantum melting of the sS crystal upon doping holes-proceeding to a uniform superfluid state via an intermediate sSS state with coexisting superflow and crystal order.

To stabilize the quarter-filled solid, we study the system with a strong nn repulsion. Figure 6 shows the results for $V_{1}=4.5$. A qS is stabilized at $\rho=1 / 4$, whereas at half-filling, a sS is formed. Doping away from quarter filling with holes, a qSS state is formed, ${ }^{29}$ as signified by the coexistence of the quarter-filled crystal structure and superfluidity. Upon further hole doping, the qSS eventually melts into a SF. Doping slightly away from quarter filling with additional bosons, we observe a similar qSS state. With further doping, however, $O_{x}$ and $O_{y}$, as well as $\rho_{s}$, begin to exhibit significant anisotropies.

Near $\mu-2 V_{1} \approx-2$, the anisotropy is most pronounced, and $S(\pi, \pi)$ vanishes, signifying a sSS state. We thus observe two seemingly unique supersolid states with different underlying crystal structures. A detailed study of the transition region in Fig. 7 indicates the presence of discontinuities developing in the structure factors and superfluid density at the transition. This indicates a first-order phase transition between the two supersolid phases, as traversed by varying the chemical potential. In a simple phenomenological defectcondensation picture, this transition may be interpreted as occurring via the first-order melting of one of the crystal 


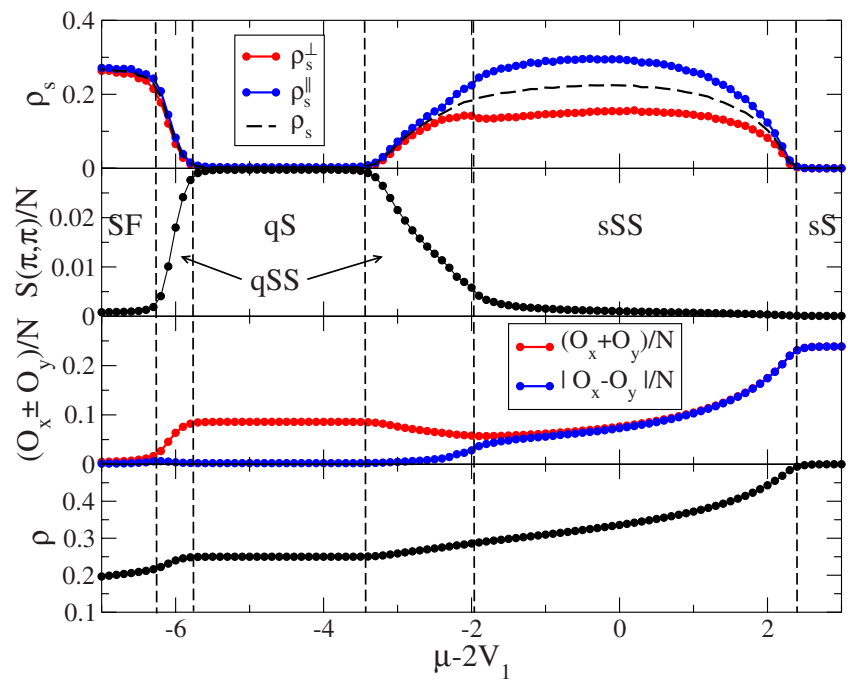

FIG. 6. (Color online) The structure factors $\rho_{s}$ and $\rho$ for $x=0.9$ and $V_{1}=4.5$. The size $L=16$ with inverse temperature $\beta=10$. Quarter-filled supersolids (qSS) and a solid (qS) are observed near $\rho=1 / 4$. A qSS to sSS transition is observed upon doping (see Fig. 7). $\rho_{s}$ also shows strong anisotropies inside the sSS phase. All quantities are in units of $t+t^{\prime}$.

sublattices that differentiate the qSS from the sSS. This interpretation is discussed more in the next section.

In Fig. 8, with slightly smaller $V_{1}=3.0$, the qSS state is still observed, yet with a reduced extent; no obvious qS crystal is observed at quarter filling on this lattice size. However, the superfluid density, although finite, shows a large dip near $\mu-2 V_{1}=2.9$ where the average particle density nears $\rho$ $=1 / 4$. In order to examine this more precisely, we performed simulations at a fixed particle density $\rho=1 / 4$ by carefully adjusting the chemical potential and restricting measurements to those Monte Carlo configurations with a particle number that precisely matches $\rho=1 / 4$. The data in Fig. 9 strongly suggests that the superfluid density indeed scales to zero in the thermodynamic limit, revealing the absence of supersolid behavior at $\rho=1 / 4$. This observation is consistent with the picture of supersolidity in this model occurring only away from commensurate crystal fillings and arising due to

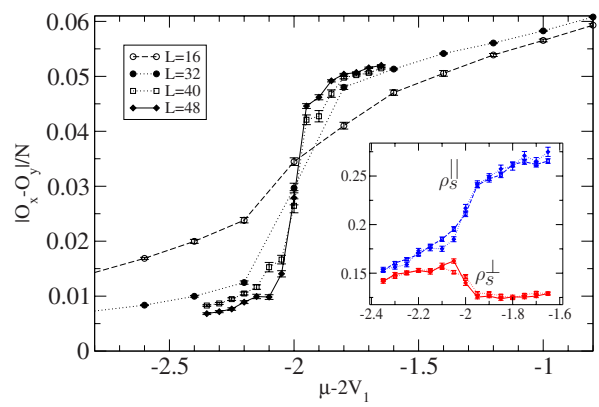

FIG. 7. (Color online) Detail of the structure factor difference (main) and superfluid density anisotropy (inset) for the simulations discussed in Fig. 6, where the discontinuities developing with increased system size indicate a first-order phase transition between the quarter-filled and striped supersolids.

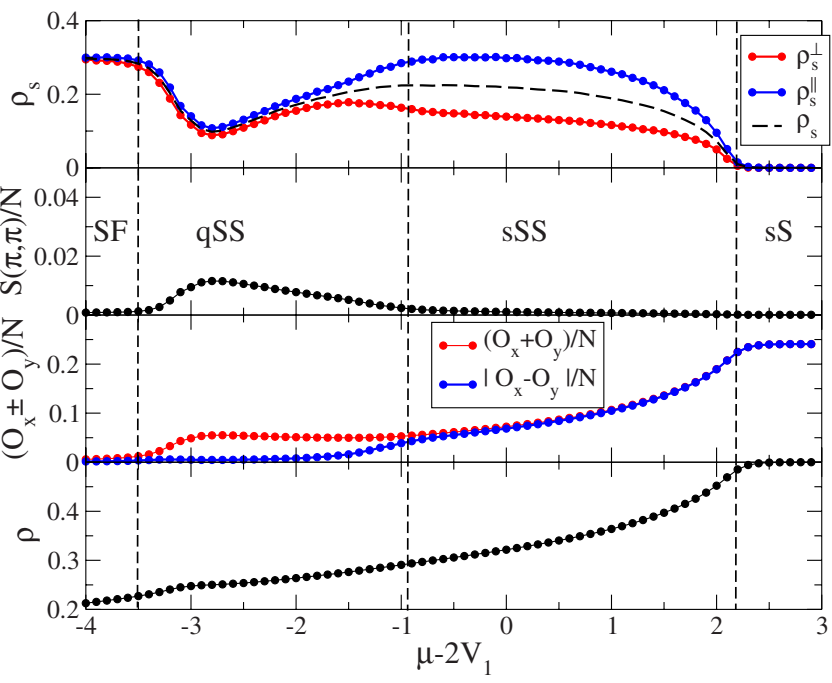

FIG. 8. (Color online) The structure factors $\rho_{s}$ and $\rho$ for $x=0.9$ and $V_{1}=3.0$. The size $L=16$ with inverse temperature $\beta=10$. All quantities are in units of $t+t^{\prime}$.

the superflow of doped defects placed interstitial to the ordered solid structures.

\section{DISCUSSION}

Using mean-field theory and quantum Monte Carlo simulations, we studied in detail the formation of three supersolid phases, which arise in the hard-core extended boson Hubbard model of Eq. (1). For large nnn repulsion, a stable checkerboard supersolid phase can be observed provided that a sufficiently strong $\mathrm{nnn}$ hopping is present, if the system is doped away from commensurate (1/2) filling. As observed in previous studies, the nn hopping itself is not sufficient to promote superflow within the doped checkerboard crystal. The other 1/2-filled crystal observed in this model is the striped solid that breaks rotational symmetry. Again, upon doping away from half-filling, a supersolid state emerges from the striped solid. Furthermore, at lower density and large repulsive interactions $\left(V_{1}\right.$ and $\left.V_{2}\right)$, the underlying density order changes to a quarter-filled crystal structure in order to avoid the large repulsions. At particle density of exactly $1 / 4$, traces of the superflow vanish.

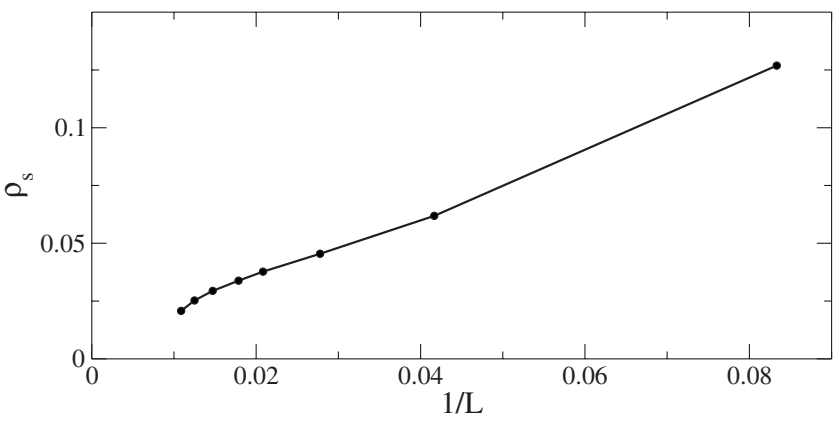

FIG. 9. Finite size behavior of $\rho_{s}$ at $\rho=1 / 4$ (see Fig. 8). The solid curve is a guide for the eyes. 
The above observations lend strong support to the idea of a mechanism for supersolidity involving the condensation of dopants (defects) outside of the lattice crystal. Indeed, in no instance, can we successfully stabilize both a finite crystal order parameter and a superfluid density at any commensurate filling. However, the simple phenomenological picture of the doped-defect condensation clearly breaks down at least for the striped supersolid phase, where although $\rho_{s}^{\perp}$ and $\rho_{s}^{\|}$show a strong anisotropy, $\rho_{s}^{\perp}$ remains finite even close to the half-filled striped crystal. This demonstrates that the superfluidity in the striped supersolid is not merely a onedimensional superflow through one-dimensional channels. This finding is similar to observations in other models on the square lattice ${ }^{17,18,28}$ and contrasts to the very weak anisotropies observed on a triangular lattice striped supersolid at half-filling. ${ }^{21}$

The presence of different supersolid phases in this model also raises the interesting possibility of observing direct supersolid-supersolid phase transitions. In particular, upon tuning $x$, we studied the intermediate region between the $t^{\prime}$ $-V_{1}$ and the $t-V_{2}$ models. We find that there is no direct transition between the checkerboard and the striped supersolid orders as $x$ is tuned-there is always a superfluid phase present when the repulsions become comparable. ${ }^{30}$ This is similar to the case at half-filling, where the superfluid emerges along the line $V_{1}=2 V_{2}$ without a direct transition between the checkerboard and striped solid, even when both $V_{1}$ and $V_{2}$ are large. ${ }^{16}$

In contrast, we find a direct transition between the qSS and sSS states in this model upon tuning $\mu-2 V_{1}$. A detailed finite size study reveals that this supersolid-supersolid phase transition is a first-order stripe melting transition. Tuning from the sSS toward the qSS by decreasing the chemical potential, an abrupt increase in the superfluid density component perpendicular to the stripe direction takes place, corresponding to a jump into the qSS crystal structure. This observation lends itself to the interpretation that, upon tra- versing this phase boundary, one of the two occupied sublattices, which contribute to the striped crystal, abruptly melts into a superfluid component, while the other remains its rigidity, and provides the underlying qSS crystal structure. It would be interesting to compare this mechanism to that observed in a supersolid-supersolid phase transition on the triangular lattice, ${ }^{22}$ where significantly stronger first-order behavior is observed. There have also been proposed more exotic mechanisms, where superfluids transition into nonuniform solid phases at commensurate filling, which may be compared to the current work. ${ }^{31}$

In conclusion, we have found several ground-state phases of the hard-core extended boson Hubbard model with nn and nnn hoppings and repulsions on the square lattice. Most notable, we find that supersolid states readily emerge when doped away from commensurability "near" their associated crystal phases, which sufficient kinetic (hopping) freedom is provided. The model thus proves an ideal playground for future study of concepts related to doping and the formation of supersolidity through the mechanism of condensed defects. Further studies are necessary to understand the detailed nature of the transitions between these different solid, superfluid, and supersolid phases, as well as their finite temperature properties.

\section{ACKNOWLEDGMENTS}

This work was supported by NSC and NCTS of Taiwan (Y.C.C. and Y.-J.K.), the U.S. Department of Energy, contract DE-AC05-00OR22725 with Oak Ridge National Laboratory, managed by UT-Battelle, LLC (R.G.M.), the German Research Foundation, NIC Jülich and HLRS Stuttgart (S.W.), and the National Science Foundation under Grant No. NSF PHYS05-51164 (S.W. and Y.-J.K.). R.G.M. would like to thank the Center of Theoretical Sciences and Department of Physics, National Taiwan University, for the hospitality extended during a visit, and S.W. and Y.-J.K. acknowledge hospitality of the Kavli Institute for Theoretical Physics at Santa Barbara. *yjkao@phys.ntu.edu.tw

${ }^{1}$ O. Penrose and L. Onsager, Phys. Rev. 104, 576 (1956).

${ }^{2}$ A. Andreev and I. Lifshits, Sov. Phys. JETP 29, 1107 (1969).

${ }^{3}$ G. V. Chester, Phys. Rev. A 2, 256 (1970).

${ }^{4}$ A. J. Leggett, Phys. Rev. Lett. 25, 1543 (1970).

${ }^{5}$ E. Kim and M. H. W. Chan, Nature (London) 427, 225 (2004).

${ }^{6}$ D. M. Ceperley and B. Bernu, Phys. Rev. Lett. 93, 155303 (2004).

${ }^{7}$ N. Prokof'ev and B. Svistunov, Phys. Rev. Lett. 94, 155302 (2005).

${ }^{8}$ E. Burovski, E. Kozik, A. Kuklov, N. Prokofev, and B. Svistunov, Phys. Rev. Lett. 94, 165301 (2005).

${ }^{9}$ E. Kim and M. H. W. Chan, Phys. Rev. Lett. 97, 115302 (2006).

${ }^{10}$ Ann Sophie C. Rittner and J. D. Reppy, Phys. Rev. Lett. 97, 165301 (2006).

${ }^{11}$ J. Day and J. Beamish, Phys. Rev. Lett. 96, 105304 (2006).

${ }^{12}$ M. H. Anderson et al., Science 269, 198 (1995).

${ }^{13}$ M. Greiner et al., Nature (London) 415, 39 (2002).
${ }^{14}$ A. Griesmaier, J. Werner, S. Hensler, J. Stuhler, and T. Pfau, Phys. Rev. Lett. 94, 160401 (2005).

${ }^{15}$ D. Jaksch, C. Bruder, J. I. Cirac, C. W. Gardiner, and P. Zoller, Phys. Rev. Lett. 81, 3108 (1998); K. Góral, L. Santos, and M. Lewenstein, ibid. 88, 170406 (2002); V. W. Scarola and S. Das Sarma, ibid. 95, 033003 (2005).

${ }^{16}$ G. G. Batrouni, R. T. Scalettar, G. T. Zimanyi, and A. P. Kampf, Phys. Rev. Lett. 74, 2527 (1995).

${ }^{17}$ P. Sengupta, L. P. Pryadko, F. Alet, M. Troyer, and G. Schmid, Phys. Rev. Lett. 94, 207202 (2005).

${ }^{18}$ F. Hébert, G. G. Batrouni, R. T. Scalettar, G. Schmid, M. Troyer, and A. Dorneich, Phys. Rev. B 65, 014513 (2001).

${ }^{19}$ S. Wessel and M. Troyer, Phys. Rev. Lett. 95, 127205 (2005); D. Heidarian and K. Damle, ibid. 95, 127206 (2005); R. G. Melko, A. Paramekanti, A. A. Burkov, A. Vishwanath, D. N. Sheng, and L. Balents, ibid. 95, 127207 (2005); M. Boninsegni and N. Prokof'ev, ibid. 95, 237204 (2005).

${ }^{20}$ S. Wessel, Phys. Rev. B 75, 174301 (2007); J.-Y. Gan et al., ibid. 
75, 214509 (2007).

${ }^{21}$ R. G. Melko, A. Del Maestro, and A. A. Burkov, Phys. Rev. B 74, 214517 (2006).

${ }^{22}$ S. R. Hassan, L. de Medici, and A.-M. Tremblay, Phys. Rev. B 76, 144420 (2007).

${ }^{23}$ G. G. Batrouni and R. T. Scalettar, Phys. Rev. Lett. 84, 1599 (2000).

${ }^{24}$ A. W. Sandvik and J. Kurkijärvi, Phys. Rev. B 43, 5950 (1991).

${ }^{25}$ O. F. Syljuasen and A. W. Sandvik, Phys. Rev. E 66, 046701
(2002).

${ }^{26}$ G. Schmid, Ph.D. thesis, ETH, Zürich, 2004.

${ }^{27}$ M. C. Gutzwiller, Phys. Rev. Lett. 10, 159 (1963).

${ }^{28}$ G. Schmid and M. Troyer, Phys. Rev. Lett. 93067003 (2004).

${ }^{29}$ Note, that the qSS is close to but not exactly at $\rho=1 / 4$.

${ }^{30}$ Y. C. Chen, Master thesis, National Taiwan University, Taipei, 2007.

${ }^{31}$ L. Balents, L. Bartosch, A. Burkov, S. Sachdev, and K. Sengupta, Phys. Rev. B 71, 144508 (2005); 71, 144509 (2005). 